\title{
Jardins de formigas: qual o estado do conhecimento sobre essas interações mutualísticas entre formigas e plantas?
}

\section{Ant-gardens: what is the current state of the knowledge about these mutualistic interactions between ants and plants?}

\author{
Ricardo Eduardo Vicentel (D) | Ivone Vieira da Silval (D) | Thiago Junqueira Izzo"l (1) \\ Universidade do Estado de Mato Grosso. Alta Floresta, Mato Grosso, Brasil \\ "Universidade Federal de Mato Grosso. Cuiabá, Mato Grosso, Brasil
}

\begin{abstract}
Resumo: Entre as interações mutualísticas envolvendo formigas e plantas, o exemplo mais complexo conhecido chama-se jardins de formigas. Nesta interação, as formigas constroem o ninho na vegetação, plantam sementes de epíftas e as protegem, quando crescem. Em troca, as epíftas controlam a umidade do ninho, oferecem estrutura para o seu crescimento e alimento para as formigas. Neste artigo, contextualizamos e situamos o leitor quanto à interação mutulística denominada de jardins de formigas, compilamos informações disponíveis na literatura e trazemos dados de pesquisas ainda não publicadas, mas em andamento, sobre este assunto.
\end{abstract}

Palavras-chave: Camponotus femoratus. Crematogaster levior. Dominância. Mutualismo. Parabiose.
Abstract: The most complex mutualistic interaction between ants and plants is called Ant-garden. In this interaction, ants build their nests in tree branches, plant epiphyte seeds and protect these plants as they grow. In exchange, epiphytes control the moisture in ant nests, and provide structure for nest growth and food for ants. Herein, we provide information about Ant-garden mutualistic interactions, including the currently available literature, and report about the current research being performed on this topic.

Keywords: Camponotus femoratus. Crematogaster levior. Dominance. Mutualism. Parabiosis.

\footnotetext{
VICENTE, R. E., I. V. SILVA \& T. J. IZZO, 2020. Jardins de formigas: qual o estado do conhecimento sobre essas interações mutualísticas entre formigas e plantas? Boletim do Museu Paraense Emílio Goeldi. Ciências Naturais 15(1): 55-63. DOI: http://doi.org/10.46357/ bcnaturais.v15i1.236.

Autor para correspondência: Ricardo Eduardo Vicente. Universidade do Estado de Mato Grosso (UNEMAT). Centro de Pesquisas e Tecnologia na Amazônia (CEPTAM). Laboratório de Anatomia Vegetal. Alta Floresta, MT, Brasil. CEP 78580-000 (ricardomyrmex@gmail.com).

Recebido em 14/12/2019

Aprovado em 21/02/2020

Responsabilidade editorial: Rony Peterson Santos Almeida
} 


\section{JARDINS DE FORMIGAS: UMA INTERAÇÃO MUTUALÍSTICA ENTRE FORMIGA-PLANTA}

As formigas (Hymenoptera, Formicidae) são insetos altamente abundantes e diversificados tanto em termos de espécies quanto em relação a grupos funcionais (Fittkau \& Klinge, 1973; Hölldobler \& Wilson, 1990; Baccaro et al., 2015; Santos-Silva et al., 2016; Vicente et al., 2016). Desempenham várias funções no ambiente e, por esse motivo, são consideradas engenheiras ecossistêmicas (Folgarait, 1998; Meyer et al., 2011). Elas também favorecem a diversidade de organismos, por estarem envolvidas em múltiplos tipos de interações com diversos outros animais (Kaminski et al., 2009; Gallego-Ropero et al., 2013; Puker et al., 2015), inclusive com outras formigas (Gallego-Ropero \& Feitosa, 2014; Rabeling et al., 2019), microrganismos (Nepel et al., 2016; Sanders et al., 2014; Mueller et al., 2017) e plantas (Frederickson, 2005; Nepel et al., 2016; Dáttilo et al., 2009; Izzo \& Benelli-Petini, 2011).

As interações desempenhadas por esses insetos com outros organismos apresentam-se variadas dentro do contexto das relações ecológicas conhecidas, podendo ser prejudiciais ou benéficas mutuamente (Frederickson, 2005; Dáttilo et al., 2009; Cerdá \& Dejean, 2011; Rabeling et al., 2019). Dentre essas interações, destaque-se as que são cooperativas entre as espécies como um dos temas centrais em ecologia, principalmente o mutualismo (Bronstein, 2001). Nesta interação, uma espécie realiza uma tarefa ou fornece um recurso que a outra espécie não consegue executar ou adquirir sozinha e recebe um tipo de pagamento ou gratificação pelo benefício desempenhado (Hoeksema \& Bruna, 2000).

Considerando-se a diversidade de interações mutualísticas entre formigas e plantas (Dáttilo et al., 2009), as plantas mais estudadas são as que possuem nectários extraflorais (Del-Claro et al., 2016; Lange et al., 2013; Dáttilo et al., 2013; Heil, 2015) e as mirmecófitas, ou seja, aquelas que possuem cavidades ocas que servem de abrigo para formigas (Michelangeli, 2010; Izzo \& Benelli-Petini, 2011; Izzo \& Vasconcelos, 2002; Dejean et al., 2018a).
Há, no entanto, uma notável interação mutualística envolvendo essas espécies, encontrada em florestas tropicais, que pode reunir todas as formas de interações entre formigas e plantas citadas anteriormente (e.g. Kaufmann \& Maschwitz, 2006; Orivel \& Leroy, 2011), conhecida como jardins de formigas (JF) (Figura 1), a qual ainda é pouco estudada.

Sabe-se que muitas espécies de formigas mantêm interações facultativas com epífitas, nidificando no emaranhado de raízes dessas plantas e protegendo tanto a epífita quanto a planta suporte (Dejean \& Olmsted, 1997; DaRocha et al., 2016). Em jardins de formigas, no entanto, a interação não é facultativa, pois as formigas constroem seus ninhos e plantam epíitas específicas, onde constantemente incorporam matéria orgânica para o crescimento das plantas (Kleinfeldt, 1978; Davidson, 1988; Kaufmann et al., 2001; Youngsteadt et al., 2009; Weissflog et al., 2017; MoralesLinares et al., 2018), sendo a principal responsável pela dispersão de sementes dessas espécies vegetais (Youngsteadt et al., 2009). Cerca de 0,67\% das espécies que ocorre na região paleotropical ocorre em JF, e essa porcentagem é ainda menor na região neotropical (menos de 0,35\%), onde a diversidade biológica é maior. Esse número cai pela metade, aproximadamente, quando considerada a quantidade de espécies de epífitas restritas a JF (Orivel \& Leroy, 2011). Apesar do conhecimento atual sobre a florística dos JF, ainda não se sabe se a dispersão é o único limitante para essas plantas ou se existem outros fatores envolvidos.

Com o estabelecimento e o crescimento das epífitas e, consequentemente, o adensamento das raízes, que controlam a umidade do ninho (Yu, 1994), as formigas podem expadir os ninhos utilizando as raízes como vigas (R. E. Vicente, observação pessoal, 2020). Ao crescerem, essas espécies emitem biomassa vegetal fresca, a qual é atrativa para os herbívoros, sendo que as formigas protegem as plantas, reagindo aos possíveis estímulos de predação, afastando os herbívoros e, consequentemente, reduzindo a herbivoria nestas plantas (Vantaux et al., 2007; Vicente et al., 2014; Leal et al., 2017). Em contrapartida, 


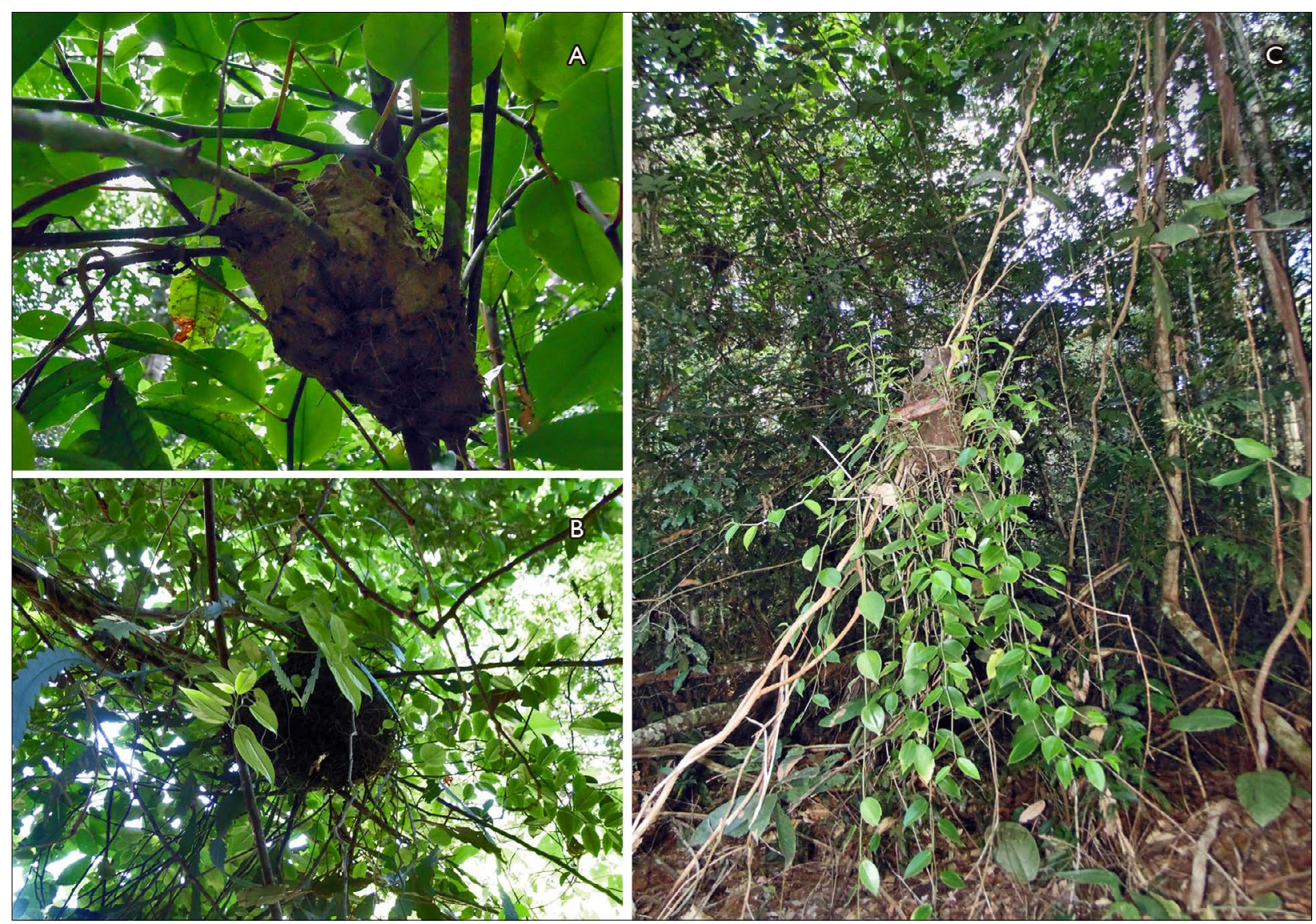

Figura 1. Jardins de formigas registrados nos módulos do Programa de Pesquisa em Biodiversidade (PPBio), localizados no município de Cláudia, Mato Grosso: A) vista lateral demonstrando as paredes de cartão com ramificações de Peperomia; B) jardim de formigas com Epiphyllum e Codonanthe ocupando um emaranhado de galhos no dossel; C) jardim coberto apenas por Peperomia crescendo em lianas ligadas ao dossel da floresta. Fotos: R. E. Vicente (2014).

com o aumento do fitness das epífitas, elas conseguem oferecer mais alimentos para as formigas através dos nectários e dos frutos, aumentando o sucesso reprodutivo dos insetos, que se tornam mais frequentes (Kleinfeldt, 1978; Davidson, 1988; Leal et al., 2017).

\section{AS ESPÉCIES ENVOLVIDAS NOS JARDINS DE FORMIGAS}

Ao longo das regiões paleo e neotropical, são conhecidas 137 espécies de epífitas e 30 de formigas habitando jardins de formigas (Orivel \& Leroy, 2011). Esse número corresponde a uma ínfima porcentagem da diversidade de espécies dos grupos ao longo do globo (Zotz, 2013;
Bolton, 2019). Apesar de compor um sistema ecológico interessante, que vai além da interação formiga-planta (Carrias et al., 2012), permanece como um sistema pobremente estudado. Exemplo dessa lacuna é a falta de conhecimento sobre a especificidade da interação tanto por parte da planta quanto da formiga, que pode ser oportunista e colonizar um JF desocupado (Kaufmann \& Maschwitz, 2006; Orivel \& Leroy, 2011).

Na região neotropical, são encontradas nove espécies de formigas e 53 de epífitas habitantes obrigatórias de JF (Orivel \& Leroy, 2011). Os JF das formigas Camponotus femoratus (Fabricius, 1804) (Formicinae) com Crematogaster levior Longino, 2003 (Myrmicinae) são os mais abundantes e

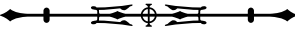


frequentes, compondo cerca de 95\% dos JF de uma área de floresta, estando presentes em cerca de 19\% das clareiras, além de apresentarem a mais ampla distribuição geográfica ocorrendo ao longo da floresta amazônica (Davidson, 1988; Youngsteadt et al., 2010; Vicente \& Izzo, 2017). Em expedições de campo realizadas entre fevereiro e maio de 2019, em uma área do sul da floresta amazônica (fazenda São Nicolau, Cotriguaçu, Mato Grosso), foram encontradas 11 clareiras com JF. Destas, seis colônias eram habitadas por $C a$. femoratus com Cr. levior, duas eram habitadas por Azteca sp., duas por Odontomachus mayi Mann, 1912 com Dolichoderus debilis Emery, 1890 e uma colônia por Odontomachus mayi com Dolichoderus decollatus F. Smith 1858. As colônias de Ca. femoratus com Cr. levior possuíam, em média, 17 ninhos (com mínimo de três e máximo de 33), enquanto as colônias das outras espécies possuíam um a dois ninhos. As colônias de Ca. femoratus com Cr. levior foram encontradas distantes mais de 2 km das colônias de Azteca sp., a cerca de 50 metros das colônias das outras espécies de formigas. Essa distância entre os JF de Ca. femoratus com Cr. levior e Azteca sp. corrobora o encontrado por Dejean et al. (2018b), na Guiana Francesa, que demonstraram a ocorrência de mosaicos criados pela dominância dessas espécies. Apesar de as espécies relatadas serem encontradas sobre o mesmo JF, não há comprovações in loco ou relatos na literatura afirmando parabiose entre essas espécies, somente entre Ca. femoratus e $C$ r. levior.

\section{UM ANINHAMENTO DE INTERAÇÕES: O CASO DOS JF DE Camponotus femoratus E Crematogaster levior}

Uma vez que os JF habitados por Camponotus femoratus e Crematogaster levior são os mais comumente encontrados na região neotropical (Davidson, 1988; Orivel \& Leroy, 2011), não coincidentemente também são os mais bem conhecidos e estudados. Essa interação das formigas parabióticas com suas epífitas mutualistas é tão robusta que as epífitas mais frequentes possuem voláteis em suas sementes, facilitando o reconhecimento por sua parceira mutualista, que dispersa estas sementes
(Davidson et al., 1990; Youngsteadt et al., 2008, 2009, 2010). Como as epífitas de JF precisam de luz para crescer (Orivel \& Leroy, 2011; Leroy et al., 2017), JF destas espécies têm sido documentados principalmente em clareiras formadas por árvores caídas, margens de rios e estradas abandonadas (Davidson, 1988; Vicente et al., 2014; Paolucci et al., 2016; Leal et al., 2017; Vicente \& Izzo, 2017).

Nestes JF, Ca. femoratus e Cr. levior, além de interagirem com algumas espécies de epífitas na relação mutualista denominada de jardins de formigas, coabitam os ninhos, convivendo em parabiose, ou seja, dividem o mesmo ninho e trilhas de forrageamento (Swain, 1980; Hölldobler \& Wilson, 1990). Apesar disso, não se sabe ao certo qual das duas espécies parabióticas inicia a construção dos ninhos. É possível que seja Ca. femoratus (Orivel \& Leroy, 2011), já que ela dispersa e planta as sementes das epífitas mutualistas, além de proteger o ninho (Youngsteadt et al., 2009; Vicente et al., 2014). Esse padrão é muito similar com outro caso de parabiose em jardins de formigas envolvendo espécies de Camponotus e Crematogaster conhecido para Malásia (Menzel \& Blüthgen, 2010; Menzel et al., 2014). No caso das formigas parabióticas neotropicais, há uma tendência para a partição temporal de nicho, uma vez que $\mathrm{Cr}$. levior forrageia mais durante o período da noite, sendo observado o contrário com Ca. femoratus (Vantaux et al., 2007). Nossa experiência acumulada nos últimos dez anos trabalhando no sistema, e com base em dados coletados nas expedições de campo citadas anteriormente, permite-nos inferir que, em clareiras onde os JF das formigas parabióticas ocorrem, encontra-se um aglomerado de ninhos em várias fases de desenvolvimento dispersos em diferentes alturas, podendo os ninhos desses aglomerados ser vistos desde cerca de $30 \mathrm{~cm}$ de altura do solo até cerca de $20 \mathrm{~m}$ de altura com auxilio de binóculos (parecendo seguir o rebaixamento do dossel). Entre os ninhos, os iniciais podiam ser encontrados em ramificações de galhos, vilosidades de lianas, em coroas de palmeiras (a cerca de $30 \mathrm{~cm}$ de altura do solo), assim como em cavidades como galhos mortos, sem mudas de epífitas, onde visualmente podia ser visto apenas $C$ r. levior. 
Apesar da presença de JF estar relacionada ao tamanho da clareira florestal (Vicente \& Izzo, 2017), a composição de espécies de epífitas habitando os jardins de formigas em escala local é determinada pelo tipo de habitat e pela proximidade entre os ninhos, e não pela variação de fatores ambientais em pequenas escalas (Paolucci et al., 2016; Leal et al., 2017). Porém, em escala local, o número das epífitas de uma dada área aumenta consideravelmente se nessa área houver JF das formigas parabióticas, pois possuem a maior riqueza entre os JF (Céréghino et al., 2011). Sabe-se que as plantas possuem alelopatia química, inibindo o crescimento de outras plantas (Mongelli et al., 1997; Kremer et al., 2018), e também é conhecido o fato de que formigas podem desempenhar alelopatia ecológica cortando folhas de plantas que competem com sua hospedeira (processo conhecido como "prunning", de acordo com Frederickson, 2005). Há, no entanto, plantas do pool regional que nunca são encontradas nos jardins de formigas e não se sabe, por exemplo, se as epífitas mutualistas e as formigas agem como filtros, selecionando a composição das epífitas através de alelopatia química ou de alelopatia ecológica.

Não bastasse a diversidade de formigas e epífitas, as bromélias da espécie Aechmea mertensii (G. Mey.) Schult. \& Schult. f. possuem a maior riqueza e abundância de protistas e insetos aquáticos em seu tanque (Céréghino et al., 2011; Carrias et al., 2012). Além disso, a estabilidade do sistema permitiu a existência de outros organismos na associação, por exemplo, em alguns JF de Ca. femoratus e $\mathrm{Cr}$. levior pode ser encontrada uma terceira espécie de formiga, Solenopsis picea Emery, 1896, em um parasitismo facultativo, denominado de lestobiose (Davidson, 1988; Orivel et al., 1997; AntWiki, 2019).

Além de promoverem a diversidade de outros organismos, as formigas parabióticas aumentam o fitness das plantas mutualistas, isso porque, quando comparado a outros tipos de substratos, os de jardins de formigas, especialmente os de $\mathrm{Ca}$. femoratus e $\mathrm{Cr}$. levior, são mais ricos em nutrientes essenciais para o crescimento vegetativo e a reprodução das epífitas (Blüthgen et al., 2001). Estas formigas são, ainda, responsáveis pela manutenção dos substratos, tanto que a ausência delas pode acarretar a perda de substrato e de epífitas (Schmit-Neuerburg \& Blüthgen, 2007). Esse incremento de minerais não se resume ao substrato, pois bromélias que funcionam como tanques de JF recebem maior input de nitrogênio, trazendo maior benefício para a planta (Leroy et al., 2011). Os benefícios são tão evidentes que as bromélias que nascem nos JF das formigas parabióticas possuem cerca de quatro vezes mais flores e frutos, e estes últimos possuem cerca de uma vez e meia mais sementes e biomassa (Leroy et al., 2011). Portanto, os JF das formigas parabióticas podem ser considerados um aninhamento de interações.

\section{UM OLHAR PARA ALÉM DOS JARDINS: NEM TUDO SÃO FLORES}

No caso dos JF das formigas parabióticas, há dominância numérica e comportamental de Ca. femoratus apresentando comportamento agressivo na monopolização de recursos e na defesa do ninho (Davidson, 1988; Vasconcelos et al., 2003; Mertl et al., 2010; Vicente et al., 2014). Esta espécie chamou a atenção do proeminenente biólogo Edward Wilson, que a categorizou como uma das formigas mais agressivas do mundo (Wilson, 1987). É sugerido que Cr. levior perdeu sua defesa química em detrimento da defesa ofertada pela sua parceira parabiótica (Longino, 2003). Como, além de agressivas, estas espécies são frequentemente amostradas forrageando do solo até o dossel das florestas (Wilson, 1987; Dejean et al., 2007, 2018b, 2019; Ryder Wilkie et al., 2010; Vicente \& Izzo, 2017), é necessário saber a amplitude da dominância dentro e para além da clareira onde ocorrem. Os JF desta espécie ocorrem em clareiras ao longo da Amazônia, e tudo indica que esta espécie pode influenciar processos ecossistêmicos, com práticas como herbivoria, polinização, dispersão de sementes, podendo alterar o processo sucessional de clareiras ao longo dessa floresta. 


\section{CONCLUSÃO E PERSPECTIVAS DE PESQUISAS FUTURAS}

Como discutido neste texto, os jardins de formigas são interações robustas ao longo de sua ocorrência, e envolvem todos os tipos de interações mutualísticas entre formigas e plantas conhecidas em um único sistema. Entre os JF, os de Camponotus femoratus e Crematogaster levior parecem ser os mais complexos, além de possuírem a maior diversidade de interatores. Por serem dominantes agressivamente, numericamente e com frequência ativas, e também por ocorrerem em habitats temporários, em constante processo sucessional, essas espécies podem ser responsáveis por impactos em processos ecossistêmicos e por mosaicos coevolutivos ao longo de sua distribuição. Apesar da grandeza deste sistema, os estudos ainda são poucos, sendo a maioria breve e isolada geograficamente. Por estes motivos, futuros estudos devem investigar fatores que determinam a ocorrência local; o que promove a diversidade de interatores; o quão abrangente é o efeito desta interação tanto em questões de força modeladora da comunidade e processos ambientais, quanto em espaço de abrangência, considerando-se possíveis variações ao longo de sua ampla ocorrência.

\section{AGRADECIMENTOS}

REV agradece fortemente a ONF-Brasil, aos funcionários da fazenda São Nicolau, ao Núcleo de Estudos da Biodiversidade da Amazônia Mato-grossense (NEBAM) e a Domingos de Jesus, pelo suporte logístico. REV também agradece à Coordenação de Aperfeiçoamento de Pessoal de Nível Superior (CAPES), pelas bolsas durante a pósgraduação, ao Conselho Nacional de Desenvolvimento Científico e Tecnológico (CNPq), pelos suportes financeiros à pesquisas (processo n 479243/2012-3), e, atualmente, à Fundação de Amparo à Pesquisa de Mato Grosso (FAPEMAT - n 0602346/2017), conjuntamente ao CNPq (n० 313839/2019-0), pelo financiamento do projeto de Desenvolvimento Científico Regional (DCR-003/2016), bem como à Universidade Estadual de Mato Grosso
(UNEMAT), pelo suporte às pesquisas atuais. TJI agradece à Bolsa de Produtividade em Pesquisa do CNPq - Nível 2 (309552/2018-4).

\section{REFERÊNCIAS}

ANTWIKI, 2019. Solenopsis picea. Disponivel em: https://antwiki. org/wiki/Solenopsis picea. Acesso em: 24 novembro 2019.

BACCARO, F. B., R. M. FEITOSA, F. FERNÁNDEZ, I. O. FERNANDES, T. J. IZZO, J. L. P. SOUZA \& R. SOLAR, 2015. Guia para os gêneros de formigas do Brasil: 1-355. Editora INPA, Manaus.

BLÜTHGEN, N., V. SCHMIT-NEUERBURG, S. ENGWALD \& W. BARTHLOTT, 2001. Ants as epiphyte gardeners: comparing the nutrient quality of ant and termite canopy substrates in a Venezuelan lowland rain forest. Journal of Tropical Ecology 17(6): 887-894. DOI: https://doi.org/10.1017/S0266467401001651.

BOLTON, B., 2019. An online catalog of the ants of the world. Disponível em: http://antcat.org. Acesso em: 24 novembro 2019.

BRONSTEIN, J. L., 2001. The exploitation of mutualisms. Ecology Letters 4(3): 277-287. DOI: https://doi.org/10.1046/j.14610248.2001.00218.x.

CARRIAS, J. F., O. BROUARD, C. LEROY, R. CÉRÉGHINO, L. PÉLOZUELO, A. DEJEAN \& B. CORBARA, 2012. An ant-plant mutualism induces shifts in the protist community structure of a tank-bromeliad. Basic and Applied Ecology 13(8): 698-705. DOI: https://doi.org/10.1016/j.baae.2012.10.002.

CERDÁ, X. \& A. DEJEAN, 2011. Predation by ants on arthropods and other animals. In: C. POLIDORI (Ed.): Predation in the Hymenoptera: an evolutionary perspective: 39-78. Transworld Research Network, Kerala, India.

CÉRÉGHINO, R., C. LEROY, J. F. CARRIAS, L. PELOZUELO, C. SÉGURA, C. BOSC, A. DEJEAN \& B. CORBARA, 2011. Ant-plant mutualisms promote functional diversity in phytotelm communities. Functional Ecology 25(5): 954-963. DOI: https:// doi.org/10.1111/j.1365-2435.2011.01863.x.

DAROCHA, W. D., F. S. NEVES, W. DÁTTILO \& J. H. DELABIE, 2016. Epiphytic bromeliads as key components for maintenance of ant diversity and ant-bromeliad interactions in agroforestry system canopies. Forest Ecology and Management 372: 128-136. DOI: https://doi.org/10.1016/j.foreco.2016.04.011.

DÁTTILO, W., E. C. MARQUES, J. C. F. FALCÃO \& D. D. O. MOREIRA, 2009. Interações mutualísticas entre formigas e plantas. EntomoBrasilis 2(2): 32-36.

DÁTTILO, W., V. RICO-GRAY, D. J. RODRIGUES \& T. J. IZZO, 2013. Soil and vegetation features determine the nested pattern of ant-plant networks in a tropical rainforest. Ecological Entomology 38(4): 374-380. DOI: https://doi.org/10.1111/een.12029. 
DAVIDSON, D. W., 1988. Ecological studies of neotropical ant-gardens. Ecology 69(4): 1138-1152. DOI: https://doi. org/10.2307/1941268.

DAVIDSON, D. W. , J. L. SEIDEL \&W. EPSTEIN, 1990. Neotropical ant gardens II: bioassays of seed compounds. Journal of Chemical Ecology 16(2): 2993-3013. DOI: https://doi.org/10.1007/ BF00979490.

DEJEAN, A. \& I. OLMSTED, 1997. Ecological studies on Aechmea bracteata (Swartz) (Bromeliaceae). Journal of Natural History 31(9): 1313-1334. DOI: https://doi.org/10.1080/00222939700770741.

DEJEAN, A., B. CORBARA, J. ORIVEL \& M. LEPONCE, 2007. Rainforest canopy ants: the implications of territoriality and predatory behavior. Functional Ecosystems and Communities 1(2): 105-120.

DEJEAN, A., F. AZÉMAR, F. PETITCLERC, J. H. DELABIE, B. CORBARA, C. LEROY, R. CÉRÉGHINO \& A. COMPIN, 2018 a. Highly modular pattern in ant-plant interactions involving specialized and non-specialized myrmecophytes. The Science of Nature 105(78): 43. DOI: https://doi.org/10.1007/s00114-018-1570-0.

DEJEAN, A., J. ORIVEL, M. LEPONCE, A. COMPIN, J. H. DELABIE, F. AZÉMAR \& B. CORBARA, 2018b. Ant-plant relationships in the canopy of an Amazonian rainforest: the presence of an ant mosaic. Biological Journal of the Linnean Society 125(2): 344-354. DOI: https://doi.org/10.1093/BIOLINNEAN/BLY125.

DEJEAN, A., A. COMPIN, J. H. DELABIE, F. AZÉMAR, B. CORBARA \& M. LEPONCE, 2019. Biotic and abiotic determinants of the formation of ant mosaics in primary Neotropical rainforests. Ecological Entomology 44(4): 560-570. DOI: https://doi. org/10.1111/een.12735.

DEL-CLARO, K., V. RICO-GRAY, H. M. TOREZAN-SILINGARDI, E. ALVES-SILVA, R. FAGUNDES, D. LANGE, W. DÁTILLO, A. A. VILELA, A. AGUIRE \& D. RODRIGUEZ-MORALES, 2016. Loss and gains in ant-plant interactions mediated by extrafloral nectar: fidelity, cheats, and lies. Insectes Sociaux 63(2): 207-221. DOI: https://doi. org/10.1007/s00040-016-0466-2.

FITTKAU, E. J. \& H. KLINGE, 1973. On biomass and trophic structure of the Central Amazonian rain forest ecosystem. Biotropica 5(1): 2-14. DOI: https:/doi.org/10.2307/2989676.

FOLGARAIT, P. J., 1998. Ant biodiversity and its relationship to ecosystem functioning: a review. Biodiversity \& Conservation 7: 1221-1244. DOI: https://doi.org/10.1023/A:1008891901953.

FREDERICKSON, M. E., 2005. Ant species confer different partner benefits on two neotropical myrmecophytes. Oecologia 143(3): 387-395. DOI: https://doi.org/10.1007/s00442-004-1817-7.

GALLEGO-ROPERO, M. C., R. M. FEITOSA \& J. R. PUJOL-LUZ, 2013. Formigas (Hymenoptera, Formicidae) associadas a ninhos de Cornitermes cumulans Kollar (Isoptera, Termitidae) no Cerrado do Planalto Central do Brasil. EntomoBrasilis 6(1): 97-101. DOI: https:// doi.org/10.12741/ebrasilis.v6i1.283.
GALLEGO-ROPERO, M. C. \& R. M. FEITOSA, 2014. Evidences of batesian mimicry and parabiosis in ants of the Brazilian Savanna. Sociobiology 61(3): 281-285. DOI: https://doi.org/10.13102/ sociobiology.v61i3.281-285.

HEIL, M., 2015. Extrafloral nectar at the plant-insect interface: a spotlight on chemical ecology, phenotypic plasticity, and food webs. Annual Review of Entomology 60: 213-232. DOI: https://doi. org/10.1146/annurev-ento-010814-020753.

HOEKSEMA, J. D. \& E. M. BRUNA, 2000. Pursuing the big questions about interspecific mutualism: a review of theoretical approaches. Oecologia 125(3): 321-330. DOI: https://doi. org/10.1007/s004420000496.

HÖLLDOBLER, B. \& E. O. WILSON, 1990. The ants: 1-732. Springer, Berlim.

IZZO, T. J. \& H. L. VASCONCELOS, 2002. Cheating the cheater: domatia loss minimizes the effects of ant castration in an Amazonian ant-plant. Oecologia 133(2): 200-205. DOI: http://dx.doi. org/10.1007/s00442-002-1027-0.

IZZO, T. J. \& A. BENELLI-PETINI, 2011. Relação entre diferentes espécies de formigas e a mirmecófita Cordia nodosa Lamarck (Boraginaceae) em áreas de mata ripária na Amazônia matogrossense. Acta Amazonica 41(3): 355-360. DOI: http://dx.doi. org/10.1590/S0044-59672011000300004.

KAMINSKI, L. A., S. F. SENDOYA, A. V. FREITAS \& P. S. OLIVEIRA, 2009. Ecologia comportamental na interface formiga-plantaherbívoro: interações entre formigas e lepidópteros. Oecologia Brasiliensis 13(1): 27-44. DOI: http://dx.doi.org/10.4257/ oeco.2009.1301.03.

KAUFMANN, E., A. WEISSFLOG, R. HASHIM \& U. MASCHWITZ, 2001. Ant-gardens on the giant bamboo Gigantochloa scortechinii (Poaceae) in West-Malaysia. Insectes Sociaux 48(2): 125-133. DOI: https://doi.org/10.1007/PL00001754.

KAUFMANN, E. \& U. MASCHWITZ, 2006. Ant-gardens of tropical Asian rainforests. Naturwissenschaften 93(5): 216. DOI: https:// doi.org/10.1007/s00114-005-0081-y.

KLEINFELDT, S. E., 1978. Ant-gardens: the interaction of Codonanthe crassifolia (Gesneriaceae) and Crematogaster longispina (Formicidae). Ecology 59(3): 449-456. DOI: https:// doi.org/10.2307/1936574.

KREMER, T. C. B., O. M. YAMASHITA, I. V. SILVA, A. C. BATISTÃO, M. P. PEREIRA, M. A. C. CARVALHO \& A. M. ROCHA, 2018. Allelopathic influence of aqueous extract of Stachytarpheta cayennensis (Rich.) Vahl on seed germination and initial seedling growth of Cucumis sativus L. International Journal of Plant \& Soil Science 26(3): 1-11. DOI: https://doi.org/10.9734/ IJPSS/2018/46525. 
LANGE, D., W. DATTILO \& K. DEL-CLARO, 2013. Influence of extrafloral nectary phenology on ant-plant mutualistic networks in a neotropical savanna. Ecological Entomology 38(5): 463-469. DOI: https://doi.org/10.1111/een.12036.

LEAL, L. C., C. C. JACOVAK, P. E. D. BOBROWIEC, J. L. C. CAMARGO \& P. E. C. PEIXOTO, 2017. The role of parabiotic ants and environment on epiphyte composition and protection in ant gardens. Sociobiology 64(3): 276-283. DOI: https://doi. org/10.13102/sociobiology.v64i3.1219.

LEROY, C., B. CORBARA, L. PÉLOZUELO, J. F. CARRIAS, A. DEJEAN \& R. CÉRÉGHINO, 2011. Ant species identity mediates reproductive traits and allocation in an ant-garden bromeliad. Annals of Botany 109(1): 145-152. DOI: https://doi.org/10.1093/ aob/mcr253.

LEROY, C., F. PETITCLERC, J. ORIVEL, B. CORBARA, J. F. CARRIAS, A. DEJEAN \& R. CÉRÉGHINO, 2017. The influence of light, substrate and seed origin on the germination and establishment of an ant-garden bromeliad. Plant Biology 19(1): 70-78. DOI: https://doi.org/10.1111/plb.12452.

LONGINO, J. T., 2003. The Crematogaster (Hymenoptera, Formicidae, Myrmicinae) of Costa Rica. Zootaxa 151(1): 1-150. DOI: https://doi.org/10.11646/zootaxa.151.1.1.

MENZEL, F. \& N. BLÜTHGEN, 2010. Parabiotic associations between tropical ants: equal partnership or parasitic exploitation? Journal of Animal Ecology 79(1): 71-81. DOI: https://doi. org/10.1111/j.1365-2656.2009.01628.x.

MENZEL, F., H. KRIESELL \& V. WITTE, 2014. Parabiotic ants: the costs and benefits of symbiosis. Ecological Entomology 39(4): 436-444. DOI: https://doi.org/10.1111/een.12116.

MERTL, A. L., M. D. SORENSON \& J. F. A. TRANIELLO, 2010. Community-level interactions and functional ecology of major workers in the hyperdiverse ground-foraging Pheidole (Hymenoptera, Formicidae) of Amazonian Ecuador. Insectes Sociaux 57(4): 441-452. DOI: https://doi.org/10.1007/s00040010-0102-5.

MEYER, S. T., I. R. LEAL, M. TABARELLI \& R. WIRTH, 2011. Ecosystem engineering by leaf-cutting ants: nests of Atta cephalotes drastically alter forest structure and microclimate. Ecological Entomology 36(1): 14-24. DOI: https://doi.org/10.1111/j.13652311.2010.01241.x.

MICHELANGELI, F. A., 2010. Neotropical myrmecophilous Melastomataceae: an annotated list and key. Proceedings of the California Academy of Sciences 61(9): 409-449.

MONGELLI, E., C. DESMARCHELIER, J. COUSSIO \& G. CICCIA, 1997. The potential effects of allelopathic mechanisms on plant species diversity and distribution determined by the wheat rootlet growth inhibition bioassay in South American plants. Revista Chilena de Historia Natural 70: 83-89.
MORALES-LINARES, J., J. G. GARCÍA-FRANCO, A. FLORESPALACIOS, J. E. VALENZUELA-GONZÁLEZ, M. MATA-ROSAS \& C. DÍAZ-CASTELAZO, 2018. Orchid seed removal by ants in Neotropical ant-gardens. Plant Biology 20(3): 525-530. DOI: https://doi.org/10.1111/plb.12715.

MUELLER, U. G., H. D. ISHAK, S. M. BRUSCHI, C. C. SMITH, J. J. HERMAN, S. E. SOLOMON, A. S. MIKHEYEV, C. RABELING, J. J. SCOTT, M. COOPER, A. RODRIGUES, A. ORTIZ, C. R. F. BRANDÃO, J. E. LATTKE, F. C. PAGNOCCA, S. A. REHNER, T. R. SCHULTZ, H. L. VASCONCELOS, R. M. M. ADAMS, M. BOLLAZZI, R. M. CLARK, A. G. HIMLER, J. S. LAPOLLA, I. R. LEAL, R. A. JOHNSON, F. ROCESS, J. SOSA-CALVO, R. WIRTH \& M. BACCI JR., 2017. Biogeography of mutualistic fungi cultivated by leafcutter ants. Molecular Ecology 26(24): 6921-6937. DOI: https://doi.org/10.1111/mec.14431.

NEPEL, M., H. VOGLMAYR, R. BLATRIX, J. T. LONGINO, K. FIEDLER, J. SCHÖNENBERGER \& V. E. MAYER, 2016. Antcultivated Chaetothyriales in hollow stems of myrmecophytic Cecropia sp. trees - diversity and patterns. Fungal Ecology 23: 131-140. DOI: https://doi.org/10.1016/j.funeco.2016.07.007.

ORIVEL, J., C. ERRARD \& A. DEJEAN, 1997. Ant gardens: interspecific recognition in parabiotic ant species. Behavioral Ecology and Sociobiology 40(2): 87-93. DOI: https://doi.org/10.1007/ s002650050319.

ORIVEL, J. \& C. LEROY, 2011. The diversity and ecology of ant gardens (Hymenoptera: Formicidae; Spermatophyta: Angiospermae). Myrmecological News 14: 73-85.

PAOLUCCI, L. N., R. R. SOLAR \& L. C. LEAL, 2016. Proximity shapes similarity in epiphytic composition of Neotropical ant gardens. Journal of Tropical Ecology 32(4): 325-329. DOI: https://doi. org/10.1017/S0266467416000298.

PUKER, A., C. S. ROSA, J. OROZCO, R. R. SOLAR \& R. M. FEITOSA, 2015. Insights on the association of American Cetoniinae beetles with ants. Entomological Science 18(1): 21-30. DOI: https:// doi.org/10.1111/ens.12085.

RABELING, C., S. MESSER, S. LACAU, I. C. NASCIMENTO, M. BACCI \& J. H. C. DELABIE, 2019. Acromyrmex fowleri: a new inquiline social parasite species of leaf-cutting ants from South America, with a discussion of social parasite biogeography in the Neotropical region. Insectes Sociaux 66(3): 435-451. DOI: https:// doi.org/10.1007/s00040-019-00705-z.

RYDER WILKIE, K. T., A. L. MERTL \& J. F. TRANIELLO, 2010. Species diversity and distribution patterns of the ants of Amazonian Ecuador. PLoS One 5(10): e13146. https://doi.org/10.1371/journal. pone.0013146.

SANDERS, J. G., S. POWELL, D. J. KRONAUER, H. L. VASCONCELOS, M. E. FREDERICKSON \& N. E. PIERCE, 2014. Stability and phylogenetic correlation in gut microbiota: lessons from ants and apes. Molecular Ecology 23(6): 1268-1283. DOI: http:// dx.doi.org/10.1111/mec.12611. 
SANTOS-SILVA, L., R. E. VICENTE \& R. M. FEITOSA, 2016. Ant species (Hymenoptera, Formicidae) of forest fragments and urban areas in a Meridional Amazonian landscape. Check List 12(3): 1-7. DOI: https://doi.org/10.15560/12.3.1885.

SCHMIT-NEUERBURG, V. \& N. BLÜTHGEN, 2007. Ant-garden epiphytes are protected against drought in a Venezuelan lowland rain forest. Ecotropica 13(2): 93-100.

SWAIN, R. B., 1980. Trophic competition among parabiotic ants. Insectes Sociaux 27(4): 377-390. DOI: https://doi.org/10.1007/ BF02223730.

VANTAUX, A., A. DEJEAN, A. DOR \& J. ORIVEL, 2007. Parasitism versus mutualism in the ant-garden parabiosis between Camponotus femoratus and Crematogaster levior. Insectes Sociaux 54(1): 95-99. DOI: https://doi.org/10.1007/s00040-007-0914-0.

VASCONCELOS, H. L., A. C. MACEDO \& J. M. VILHENA, 2003. Influence of topography on the distribution of ground-dwelling ants in an Amazonian forest. Studies on Neotropical Fauna and Environment 38(2): 115-124. DOI: https://doi.org/10.1076/snfe.38.2.115.15923.

VICENTE, R. E., W. DÁTTILO \& T. J. IZZO, 2014. Differential recruitment of Camponotus femoratus (Fabricius) ants in response to ant garden herbivory. Neotropical Entomology 43(6): 519-525. DOI: https://doi.org/10.1007/s13744-014-0245-6.

VICENTE, R. E., L. P. PRADO \& T. J. IZZO, 2016. Amazon rainforest ant-fauna of Parque Estadual do Cristalino: understory and grounddwelling Ants. Sociobiology 63(3): 894-908. DOI: https://doi. org/10.13102/sociobiology.v63i3.1043.

VICENTE, R. E. \& T. J. IZZO, 2017. Defining habitat use by the parabiotic ants Camponotus femoratus (Fabricius, 1804) and Crematogaster levior Longino, 2003. Sociobiology 64(4): 373-380. DOI: https://doi.org/10.13102/sociobiology.v64i4.1228.
WEISSFLOG, A., E. KAUFMANN \& U. MASCHWITZ, 2017. Ant gardens of Camponotus (Myrmotarsus) irritabilis (Hymenoptera: Formicidae: Formicinae) and Hoya elliptica (Apocynaceae) in Southeast Asia. Asian Myrmecology 9: e009001. DOI: https://doi. org/10.20362/am.009001.

WILSON, E. O., 1987. The arboreal ant fauna of Peruvian Amazon forests: a first assessment. Biotropica 19(3): 245-251. DOI: https:// doi.org/10.2307/2388342.

YOUNGSTEADT, E., S. NOJIMA, C. HÄBERLEIN, S. SCHULZ \& C. SCHAL, 2008. Seed odor mediates an obligate ant-plant mutualism in Amazonian rainforests. Proceedings of the National Academy of Sciences of the United States of America 105(12): 4571-4575. DOI: https://doi.org/10.1073/pnas.0708643105.

YOUNGSTEADT, E., J. A. BACA, J. OSBORNE \& C. SCHAL, 2009. Species-specific seed dispersal in an obligate ant-plant mutualism. PLoS One 4(2): e4335. DOI: https://doi.org/10.1371/journal. pone.0004335.

YOUNGSTEADT, E., P. G. BUSTIOS \& C. SCHAL, 2010. Divergent chemical cues elicit seed collecting by ants in an obligate multi-species mutualism in lowland Amazonia. PloS One 5(12): e15822. DOI: https://doi.org/10.1371/journal.pone.0015822.

YU, D. W., 1994. The structural role of epiphytes in ant gardens. Biotropica 26(2): 222-226. DOI: https://doi.org/10.2307/2388813.

ZOTZ, G., 2013. The systematic distribution of vascular epiphytes - a critical update. Botanical Journal of the Linnean Society 171(3): 453-481. DOI: https://doi.org/10.1111/boj.12010. 
